\title{
A Boltzmann-kinetical description of an MHD shock with arbitrary field inclination
}

\author{
M. Siewert and H.-J. Fahr \\ Argelander Institut für Astronomie der Universität Bonn, Abteilung f. Astrophysik und Extraterrestrische Forschung, \\ Auf dem Huegel 71, 53121 Bonn, Germany \\ e-mail: msiewert@astro.uni-bonn.de
}

Received 24 October 2007 / Accepted 27 March 2008

\begin{abstract}
Aims. We revisit the general problem of the anisotropic MHD shock for arbitrary magnetic field inclinations, where the jump conditions are underdetermined. To describe the transition region of the shock, we derive a variant of a kinetic Boltzmann-Vlasov equation previously used to describe the perpendicular shock in the absence of dissipative processes.

Methods. We derive effective force terms, for the kinetic equation, that are based on the conservation of the Chew-Goldberger-Low (CGL) MHD invariants which appear in the standard model for anisotropic MHD. This approach is based on a generalisation of the well-known equivalence between the first CGL invariant and the integral over the magnetic moments of the underlying particles.

Results. Assuming an arbitrary distribution function on the upstream side, we integrate the kinetic equation across the shock. This result allows us to establish further relations between the MHD velocity moments on both sides. Using this additional information, we close the anisotropic MHD jump conditions. In addition, the now unique solution of the jump conditions allows us to present explicit cuts through a representative Maxwellian distribution function on both sides of the shock. In the kinetic equation, one only requires two parameters that need to be derived from the classical jump conditions, the classical MHD compression ratio and an equivalent ratio for the magnetic field strengths.
\end{abstract}

Key words. plasmas - shock waves - magnetohydrodynamis (MHD) - sun: solar wind

\section{Introduction}

We published previously several kinetic studies of an ion plasma crossing an MHD shock, such as the bow shock of the Earth or the solar wind termination shock (Fahr \& Siewert 2006; Siewert \& Fahr 2007a,b), that attempted to improve our understanding of this area of magnetohydrodynamics. Essentially, MHD shocks have been described using one of the following approaches, each of which possesses its own, inherent flaws.

First, from the theoretical MHD side, there are Rankine-Hugoniot-like jump conditions, based on the conservation of moments such as the MHD mass and momentum fluxes, the energy flux, and the conservation of the Poynting vector flux (see, e.g. Hudson 1970; Erkaev et al. 2000; Vogl et al. 2003). This approach is based on MHD and describes only a few low-order velocity moments of the plasma flow instead of the full ion velocity distribution function $f(\boldsymbol{w})$. Therefore, this approach is well suited to the analysis of Maxwell-Boltzmannlike distribution functions, where the entire function can be parameterised using only a few velocity moments, but may be inappropriate for, say, power-law distribution functions, as those found in most cosmic ray spectra (see, e.g. Schlickeiser 2002; Fisk \& Gloeckler 2006). In addition, it turns out that, for an anisotropic plasma, the jump conditions are underdetermined, where one possible parameterisation is to assume that the downstream pressure anisotropy, $\lambda_{2}=p_{\perp, 2} / p_{\|, 2}$, is the free parameter (Erkaev et al. 2000). Since an anisotropic plasma may emerge in the presence of magnetic fields (Chew et al. 1956), this restriction can be traced back to the inherent MHD flaw, where one initially obtains an infinite hierarchy of moment equations (see e.g. Cercignani 1988), which needs to be truncated at an arbitrary point.

The second approach to this problem is based on experimental observations, followed by appropriate modelling of these data. The cluster mission (Escoubet et al. 1997) has observed the Earth bow shock for many years now, observing its highly nonstationary behaviour (see e.g. Lobzin et al. 2007). Another prominent set of shock-related data was taken by the Voyager 1 spacecraft, which, in late 2004, crossed the solar wind termination shock (Stone et al. 2005; Decker et al. 2005): the spacecraft observed power-law spectra of practically unchanging power indices across the shock (Cummings et al. 2006), in addition to a series of magnetic bumps and holes in the heliopause (Burlaga et al. 2006a,b), which could be explained in terms of plasma waves. Up to now, however, these experimental data can only be fitted using by an ad hoc model because a robust physical explanation of their origin does not exist yet.

A third approach to the understanding of understand MHD shocks has also emerged, that is based on numerical shock simulation models completed using powerful supercomputers (e.g. Hada et al. 2003; Scholer et al. 2003): this, in principle, allows to study not only the behaviour of the individual particles, but also the nonstationarity in the "fine structure" of the transition region (i.e. fields and distribution functions inside the shock). However, numerical simulations always require the introduction of boundary conditions, in addition to a numerically stable "stepping scheme", which, in principle, may introduce additional, unphysical terms in the underlying equations (see, e.g. Press 1987-2002, Chap. 19). A consistent description of the physical boundary conditions typically requires the simulation of a 
system that is much larger than the transition region of the shock itself, and resolving this region can be difficult. One example of such a situation is the heliospheric termination shock, where the heliosphere with an average radius of the order of $100 \mathrm{AU}$ requires a grid size that is considerably different from the typical size of a shock, which is assumed to be of the order of a few gyroradii (Scholer et al. 2003; Fahr \& Siewert 2007). For these reasons, in any large-scale simulations, it is possible to verify only the existence of a shock by using the RankineHugoniot jump conditions. Results from such numerical simulations demonstrate a highly nonstationary behaviour, similar to in situ space observations, for which, however, no complete theoretical understanding exists.

Since all of these approaches are, in one way or another, incomplete, we developed an approach based on a kinetic Boltzmann-Vlasov equation, which might be able to fill the gaps between the competing descriptions. In contrast to common MHD, our approach provides a description of the entire distribution function $f(\boldsymbol{w})$, which includes all forms of nonthermal distribution functions. So far, this model allowed us to explain the conserved power-law index observed by the Voyager 1 spacecraft (Siewert \& Fahr 2007a,b), as well as the magnetic bumps and holes observed by the same spacecraft in the heliosheath (Burlaga et al. 2006b; Fahr \& Siewert 2007). Our results imply that, to obtain a (quasi-)stationary transition region, one requires additional physical processes in addition to the deceleration of ions and the change in electric and magnetic fields across the shock. Since, in our approach, the conservation of the mass flow transforms into a set of specific mathematical conditions, the additional physical processes to be included must be of a rather specific form as well, unless the transition region is highly nonstationary (Siewert \& Fahr 2007b). This result is agrees with the nonstationarity emerging in both numerical approaches and experimental observations, which also implies that additional, internal microphysics should be taken into account. In this study, we restrict ourselves to the solar wind termination shock, which is located approximately at a similar spatial position over a significant period of time. We reinvestigate our kinetic model of the MHD shock, and derive a new, improved form of the kinetic Boltzmann equation for the general shock, which is based on a new, systematic connection between the MHD view and the per-particle view of the shock. These arguments enable to be removed most of the physical and mathematical problems that emerged in previous studies (e.g. Fahr \& Siewert 2006; Siewert \& Fahr 2007a,b). As a side-result, we prove the equivalence of MHD invariants and single particle invariants, by generalising the conservation of the "magnetic CGL-moments".

\section{The kinetic approach}

\subsection{The mathematical and physical decomposition of the Boltzmann equation}

In Siewert \& Fahr (2007b), we proved that the specific form of the Boltzmann equation which was used in earlier studies is inherently unable to describe the parallel MHD shock in the anisotropic CGL model. For this reason, we now rederive the Boltzmann equation, looking carefully for shortcomings and flaws in the up-to-now description, including missing terms.

We consider a more general form of the Boltzmann equation that we later develop to include nontrivial terms, such as wave-turbulence generation, diffusion and an exchange of particle number, energy and momentum between multiple particle populations, which occur when we consider processes such as charge exchange, ionization or wave-particle induced friction. Following the earlier studies of Fahr \& Siewert (2006) and Siewert \& Fahr (2007a,b), a sufficiently general form of the Boltzmann-Vlasov equation is given by

$$
\begin{aligned}
A_{\mathrm{s}}(\boldsymbol{w}, s) \frac{\mathrm{d}}{\mathrm{d} s} f(\boldsymbol{w}, s) & =A_{\|}(\boldsymbol{w}, s) \frac{\mathrm{d}}{\mathrm{d} w_{\|}} f(\boldsymbol{w}, s) \\
& +A_{\perp}(\boldsymbol{w}, s) \frac{\mathrm{d}}{\mathrm{d} w_{\perp}} f(\boldsymbol{w}, s),
\end{aligned}
$$

where the basic Boltzmann equation has been specialised to a spatially one-dimensional problem with the streamline coordinate $s$. The coefficients $A_{\|}$and $A_{\perp}$ are, at this point, free functions, which need to be derived from the physical processes in the transition region of the shock.

In a more general approach, one has to consider small fluctuations, in terms of plasma waves and turbulence. In the presence of a stationary background magnetic field, these processes are described traditionally using the Fokker-Planck equation (see, e.g. Schlickeiser 1989; Chalov \& Fahr 1998), which then introduces terms such as

$$
\frac{1}{w^{2}} \frac{\mathrm{d}}{\mathrm{d} w}\left(w^{2} D_{w w} \frac{\mathrm{d}}{\mathrm{d} w} f\right) .
$$

Obviously, these terms lead to the emergence of second-order derivatives in Eq. (1),

$$
\begin{aligned}
A_{\mathrm{s}}(\boldsymbol{w}, s) \frac{\mathrm{d}}{\mathrm{d} s} f(\boldsymbol{w}, s)= & A_{\|}(\boldsymbol{w}, s) \frac{\mathrm{d}}{\mathrm{d} w_{\|}} f(\boldsymbol{w}, s) \\
& +A_{\perp}(\boldsymbol{w}, s) \frac{\mathrm{d}}{\mathrm{d} w_{\perp}} f(\boldsymbol{w}, s) \\
& +A_{\|,\|}(\boldsymbol{w}, s) \frac{\mathrm{d}^{2}}{\mathrm{~d} w_{\|}^{2}} f(\boldsymbol{w}, s) \\
& +A_{\perp, \perp}(\boldsymbol{w}, s) \frac{\mathrm{d}^{2}}{\mathrm{~d} w_{\perp}^{2}} f(\boldsymbol{w}, s) \\
& +A_{\|, \perp}(\boldsymbol{w}, s) \frac{\mathrm{d}^{2}}{\mathrm{~d} w_{\|} \mathrm{d} w_{\perp}} f(\boldsymbol{w}, s),
\end{aligned}
$$

as well as to further contributions to $A_{\|}$and $A_{\perp}$. For this reason, we call the coefficients $A_{i}$ the first-order and second-order Fokker-Planck terms (which are not to be confused with the better known Fokker-Planck coefficients). However, in this study, we focus on the more basic situation of a wave-free plasma, where all second-order terms vanish, since in this special situation, the general form of the solution is already known (see Siewert \& Fahr 2007b, with the main mathematical methods repeated later in this paper). Finally, we work with the gyroaveraged approach commonly found in literature, i.e. there are only two velocity components, the parallel velocity $w_{\|}$, and the perpendicular velocity $w_{\perp}$. Depending on the specific structure inside the transition region, this approach may be no longer justified; however, a more specific description of this configuration does not yet exist in an analytical approximation.

The Boltzmann equation may also be parameterised from a physical standpoint that emphasizes not the mathematical form but the physics behind it. We write this alternate representation in the form

$$
\begin{aligned}
L_{\mathrm{kin}}[f] & +L_{\mathrm{F}}[f] \\
& +L_{\mathrm{acc}}[f] \\
& +Q_{\text {source }}\left[f, f^{*}\right]=0 .
\end{aligned}
$$


Here $L_{i}$ is a linear differential operator acting on the distribution function; the subscripts stand for kinetic terms on the individual particle level, "true" Force terms, and pseudo forces within an accelerated reference frame. Finally, we take into account of a possible source/sink term, which represents a possible exchange of particles, energy, and momentum between different particle populations; this term may be, in principle, nonlinear and dependent on multiple distribution functions, such as ions and electrons. We denote the distribution function related to this additional population with an asterisk. In principle, the corresponding operator does not need to be linear, and is referred as $Q$.

\subsection{The Fokker-Planck terms in the various reference frames}

A general formulation of the collisionless Boltzmann equation (i.e. the Vlasov equation) in an accelerated reference frame is given by

$\left(\boldsymbol{w} \cdot \boldsymbol{\nabla}_{x}\right) f+\left(\boldsymbol{F} \cdot \boldsymbol{\nabla}_{w}\right) f+\left(\frac{\mathrm{d} \boldsymbol{U}}{\mathrm{d} t} \cdot \boldsymbol{\nabla}_{w}\right) f=0$,

where $\mathrm{d} \boldsymbol{U} / \mathrm{d} t$ is the acceleration of the bulk plasma system that moves with the bulk velocity $\boldsymbol{U}$, i.e. this term may be identified with $L_{\text {acc }}$. The first term of the left-hand side of the expression corresponds to the kinetic term $L_{\text {kin }}$, while the middle term may be identified with the electromagnetic force term $L_{\mathrm{F}, \mathrm{em}}$. Specializing to a one-dimensional problem, we obtain

$L_{\mathrm{kin}}[f](\boldsymbol{w}, s)=w_{\|} \cos \Theta_{B \mathrm{n}} \frac{\mathrm{d}}{\mathrm{d} s} f\left(w_{\|}, w_{\perp}\right)$

in the (accelerated) plasma frame, where $\Theta_{B \mathrm{n}}$ is the angle between the magnetic field and the shock normal. Since this is the only term containing spatial gradients of the distribution function, one obtains easily the final form of $A_{\mathrm{s}}$,

$A_{\mathrm{s}}=w_{\|} \cos \Theta_{B \mathrm{n}}(s)=U_{\mathrm{n}}\left(w_{\|}, s\right)$,

where we introduce the differential bulk velocity $U_{\mathrm{n}}\left(w_{\|}, s\right)$. This means that any temporal derivative appearing in the equation may be transformed into a spatial derivative using

$$
\frac{\mathrm{d}}{\mathrm{d} t}=w_{\|} \cos \Theta_{B \mathrm{n}}(s) \frac{\mathrm{d}}{\mathrm{d} s},
$$

Then, as long as the Fokker-Planck terms $A_{\|}$and $A_{\perp}$ are proportional to $\mathrm{d} / \mathrm{d} t$, is it possible to completely eliminate the factor $w_{\|} \cos \Theta_{B \mathrm{n}}(s)$. We note that this point was missing in an earlier equation derived in Fahr \& Siewert (2006), which produced a singularity under specific conditions. Deriving the "real" force term is more complicated because the electromagnetic tension forces (i.e. electric and magnetic force terms) must be considered. One possible alternative approach to this problem is

$\frac{\mathrm{d} f}{\mathrm{~d} t}=\sum_{i}\left(\frac{\mathrm{d} w_{\|}}{\mathrm{d} t}\right)_{i} \frac{\partial f}{\partial w_{\|}}+\sum_{i}\left(\frac{\mathrm{d} w_{\perp}}{\mathrm{d} t}\right)_{i} \frac{\partial f}{\partial w_{\perp}}$,

where the sums are performed over all parameters which get modified by the shock (and no explicitly time-dependent terms do appear). Unfortunately, collecting these parameters is still rather complicated, as several nontrivial points have to be taken into account. For example, in pure MHD, the electromagnetic fields are considered to be frozen into the plasma on the far upstream and downstream sides of the plasma, which implies that this should be true also for the small transition region in which pure MHD no longer holds. Thus, the behaviour of the particles inside the shock is not arbitrary, but restricted by several pseudoMHD properties.

In principle, there are two different ways to derive the factors $\mathrm{d} w_{i} / \mathrm{d} t$ in their final form. There is first the per-particle approach, where no a priori limits or averages are made, that considers a single particle, with three velocity coordinates including a gyroangle, and the full force acting on this particle. Then, one has to derive the modifications to this force caused by the local field modifications inside the shock, which in turn have to be derived from the particle behaviour inside the system. In a sufficiently general case, this system of equations is complicated and has to be solved numerically, i.e. the analytical forms of the kinetic terms cannot be derived.

The other approach is what we call the semikinetic approach. From the kinetic view, we borrow the idea of taking an individual particle (or, alternatively, a narrow region in velocity space), but we describe the force terms using MHD quantities. In other words, force terms and their corresponding energy and momentum exchange are implicitly included in MHD quantities such as the bulk velocity, the partial pressures, and the magnetic field tension. These quantities must then be parameterised in a way that is consistent with the MHD jump conditions, which leads to a consistent description of the transition region using MHD quantities only, where only a small part of the MHD parameters actually needs to be modelled. This produces, however, a complicated equation because all important MHD quantities must be represented, that is the magnetic field, partial pressures, bulk velocities, and mass density, which are all related in some way. Finally, one requires a systematic relation between the MHD quantities and the individual kinetic velocities, unless all particles react in an identical way to the shock, which would be unphysical. In the remaining part of this section, we introduce a formalism than enables this to be realised in a straightforward way.

We begin with a phenomenological motivation of this formalism. First, we would like to emphasize that, in a wide variety of physical systems, the magnetic moment of the individual particles is conserved, i.e.

$\frac{\mathrm{d}}{\mathrm{d} t} \mu \propto \frac{\mathrm{d}}{\mathrm{d} t} \frac{w_{\perp}^{2}}{B}=0$

For a broad distribution function $f(\boldsymbol{w})$, the total magnetic moment of all particles is conserved, which is given by

$\frac{\mathrm{d}}{\mathrm{d} t} \mu_{f}=\frac{\mathrm{d}}{\mathrm{d} t} \int \mathrm{d}^{3} w \frac{w_{\perp}^{2}}{B} f(\boldsymbol{w})=0$.

However, this expression may be interpreted in terms of an MHD velocity moment, i.e.

$$
\begin{aligned}
\frac{\mathrm{d}}{\mathrm{d} t} \mu_{f} & =\frac{\mathrm{d}}{\mathrm{d} t} \frac{1}{B} \int \mathrm{d}^{3} w w_{\perp}^{2} f(\boldsymbol{w}) \\
& =\frac{\mathrm{d}}{\mathrm{d} t} \frac{1}{B}\left\langle w_{\perp}^{2}\right\rangle \\
& =\frac{\mathrm{d}}{\mathrm{d} t} \frac{p_{\perp}}{B}
\end{aligned}
$$

As a generalisation of this, any individual particle invariant that is proportional to $w_{\|}^{i} w_{\perp}^{j}$ may be trivially transformed into an MHD form, simply by applying

$w_{\|}^{i} w_{\perp}^{j} \rightarrow\left\langle w_{\|}^{i} w_{\perp}^{j}\right\rangle$ 
For the magnetic moment, the corresponding MHD invariant is given by

$\frac{\mathrm{d}}{\mathrm{d} t} \frac{p_{\perp}}{B}=$ const.

Now, it is known that, in the CGL model for anisotropic MHD systems (Chew et al. 1956), one obtains two adiabatic invariants that relate the different MHD parameters with each other. These two invariants are given by

$\frac{\mathrm{d}}{\mathrm{d} t} \mathrm{CGL}_{1}=\frac{\mathrm{d}}{\mathrm{d} t} \frac{\mu_{f}}{\rho}=\frac{\mathrm{d}}{\mathrm{d} t} \frac{p_{\perp}}{B \rho}=0$,

and

$\frac{\mathrm{d}}{\mathrm{d} t} \mathrm{CGL}_{2}=\frac{\mathrm{d}}{\mathrm{d} t} \frac{p_{\|} B^{2}}{\rho^{3}}=0$.

Obviously, the first CGL invariant is given by a "normalised" form of the MHD magnetic moment.

In an inertial rest frame, the normalisation of the stationary distribution function is constant, i.e. $\dot{\rho}=0$. In our formalism, however, we are working in an accelerated reference frame, where $\dot{\rho} \neq 0$. Introducing the normalised distribution function

$f_{\text {norm }}(\boldsymbol{w})=\frac{f(\boldsymbol{w})}{\rho}$,

we see that the perpendicular pressure is given by

$p_{\perp} \propto\left\langle w_{\perp}^{2}\right\rangle \propto \int \mathrm{d}^{3} w w_{\perp}^{2} f_{\text {norm }}(\boldsymbol{w})$

and the additional factor $\rho^{-1}$ in Eq. (15) cancels out the contributions from the acceleration of the system. In other words, Eq. (13) has to be generalised to

$\frac{w_{\|}^{i} w_{\perp}^{j}}{\rho} \rightarrow \frac{\left\langle w_{\|}^{i} w_{\perp}^{j}\right\rangle}{\rho}=\left\langle w_{\|}^{i} w_{\perp}^{j}\right\rangle_{\text {norm }}$,

which is valid both in inertial and non-inertial rest frames.

Next, we demonstrate that, in the absence of stochastic processes, the opposite direction of Eq. (19) is also valid. In other words, it is possible, under certain conditions, to interpet an MHD invariant in terms of an integral over per-particle invariants. Taking, representatively, the second CGL invariant, we may write

$$
\begin{aligned}
\frac{\mathrm{d}}{\mathrm{d} t} \frac{p_{\|}}{\rho} \frac{B^{2}}{\rho^{2}} & =\frac{\mathrm{d}}{\mathrm{d} t} \int \mathrm{d}^{3} w w_{\|}^{2} \frac{B^{2}}{\rho^{2}} f_{\text {norm }}(\boldsymbol{w}) \\
= & \int \mathrm{d}^{3} w f_{\text {norm }}(\boldsymbol{w}) \frac{\mathrm{d}}{\mathrm{d} t}\left(w_{\|}^{2} \frac{B^{2}}{\rho^{2}}\right) \\
& +\frac{B^{2}}{\rho^{2}} \int w_{\|}^{2} \frac{\mathrm{d}}{\mathrm{d} t}\left(\mathrm{~d}^{3} w f_{\text {norm }}(\boldsymbol{w})\right)=0,
\end{aligned}
$$

Obviously, as soon as the second integral in this sum vanishes, the first integral vanishes as well, which requires that

$\frac{\mathrm{d}}{\mathrm{d} t}\left(w_{\|}^{2} \frac{B^{2}}{\rho^{2}}\right)=0$.

Since this approach is not restricted to the second CGL invariant alone, we may write our condition in the more general form

$$
\frac{\mathrm{d}}{\mathrm{d} t} \frac{\left\langle w_{\|}^{i} w_{\perp}^{j}\right\rangle}{\rho} \stackrel{!}{=} \int \mathrm{d}^{3} w f_{\text {norm }}(\boldsymbol{w}) \frac{\mathrm{d}}{\mathrm{d} t} \frac{w_{\|}^{i} w_{\perp}^{j}}{\rho}=0 .
$$

In Appendix A, we demonstrate in detail how Eq. (22) is valid under the conditions presented at the start of this section.

Although we do not study the physical nature of Eq. (21), we emphasize that the mostly mathematical approach to this identification requires that such an invariant must exist. In Fahr \& Siewert (2008), we identified this invariant in the solar wind, following from the divergence of the plasma stream and the corresponding Parker model for the frozen-in magnetic fields (Parker 1965). Using a general expression for the corresponding velocity modification,

$\frac{\mathrm{d}}{\mathrm{d} t} w_{\|}=-w_{\|} \frac{\boldsymbol{B} \cdot \boldsymbol{\nabla}}{B}\left(\frac{\boldsymbol{U} \cdot \boldsymbol{B}}{B}\right)$,

the reason for the resulting change of the parallel velocity component $w_{\|}$is due to the recognition of the bulk velocity gradient parallel to the magnetic field $\boldsymbol{B}$, by the particle, when it covers a path $w_{\|}$per unit time of its motion parallel to $\boldsymbol{B}$. However, evaluating this expression requires an intimate knowledge of the reaction of the frozen-in magnetic field, for which, inside the transition layer of the shock, no systematic theory yet exists.

Therefore, lacking any better description, we make the adhoc assumption that both CGL invariants are conserved inside the shock. This approach requires that the magnetic field must be changing slowly. In other words, the reorientation and condensation timescale $\tau_{\mathrm{c}}$ must be much larger than the gyration timescale,

$\tau_{\mathrm{c}}=\frac{\mathrm{d} B}{\mathrm{~d} t} / B \gg \tau_{\mathrm{gyr}}$.

Since all MHD quantities in the system are connected with each other, similar conditions must hold for the other MHD quantities appearing in the adiabatic invariants as well. From this requirement, it is automatically possible to derive another condition, namely the absence of particle-particle collisions. Since the conservation of the magnetic moment requires slow variations of all parameters, including the perpendicular particle velocities, any fast energy and momentum exchange mechanism (such as scattering) must be absent from the system, or the gyration of the individual particles would be significantly perturbed. The same requirement holds for the second CGL invariant to be valid (see e.g. Kulsrud 1983, p. 115).

Finally, we consider the Eqs. (21) and (10) to evaluate the temporal derivative and obtain the expressions

$\left.\frac{\mathrm{d} w_{\|}}{\mathrm{d} t}\right|_{\mathrm{CGL}_{2}}=w_{\|} \frac{\mathrm{d}}{\mathrm{d} t}(\ln \rho-\ln B)$

and

$\left.\frac{\mathrm{d} w_{\perp}}{\mathrm{d} t}\right|_{\mu}=\frac{w_{\perp}}{2} \frac{\mathrm{d}}{\mathrm{d} t} \ln B$

These two Eqs. (26) and (25), are sufficient to describe the system. Then, collecting our results, the Fokker-Planck terms are given by

$A_{\mathrm{s}}=w_{\|} \cos \Theta_{B \mathrm{n}}$

$A_{\|}=-w_{\|}^{2} \cos \Theta_{B \mathrm{n}} \frac{\mathrm{d}}{\mathrm{d} s}(\ln \rho-\ln B)$

$A_{\perp}=-\frac{w_{\perp}}{2} w_{\|} \cos \Theta_{B \mathrm{n}} \frac{\mathrm{d}}{\mathrm{d} s} \ln B$.

In these equations, the additional negative sign follows from the fact that, in Eq. (1), the force term was moved to the other side of the equation. From the argument following Eq. (8), the global 
factor $w_{\|} \cos \Theta_{B \mathrm{n}}$ may be removed, which allows us to write the simpler form

$$
\begin{aligned}
A_{\mathrm{s}} & =1 \\
A_{\|} & =-w_{\|} \frac{\mathrm{d}}{\mathrm{d} s}(\ln \rho-\ln B) \\
A_{\perp} & =-\frac{w_{\perp}}{2} \frac{\mathrm{d}}{\mathrm{d} s} \ln B .
\end{aligned}
$$

Then, we obtain the final form of the kinetic Boltzmann equation,

$$
\frac{\mathrm{d}}{\mathrm{d} s} f=-w_{\|} \frac{\mathrm{d}}{\mathrm{d} s}(\ln \rho-\ln B) \frac{\mathrm{d}}{\mathrm{d} w_{\|}} f-\frac{w_{\perp}}{2} \frac{\mathrm{d}}{\mathrm{d} s} \ln B \frac{\mathrm{d}}{\mathrm{d} w_{\perp}} f .
$$

This equation describes the full downstream distribution function at an MHD shock (instead of only a few, low-order velocity moments) using the assumption that the adiabatic invariants of the CGL theory also hold inside the transition region of the shock.

It it worth mentioning that this equation does not depend upon the magnetic field orientation, which is represented by the fact that no magnetic field projections $\left(B_{\mathrm{n}}\right.$ or $\left.B_{\mathrm{t}}\right)$ appears. Therefore, the terms related to this effect derived by Fahr \& Siewert (2006) must be discarded on account of mixing the semikinetic approach with the full kinetic approach. Since both approaches rely on different amounts of averaging and other approximations, a self-consistent description of the shock must not mix these different representations.

\subsection{Solutions of the improved Boltzmann equation}

In Siewert \& Fahr (2007b), we derived restrictions for the possible form of the Fokker-Planck terms $A_{i}$, based on the concept that the average parallel velocity $\left\langle w_{\|}\right\rangle$vanishes in the plasma frame. At this point, we repeat the nontrivial parts relevant for the solution of our kinetic equation. First, we emphasize that the adiabatic invariants (Eqs. (15) and (16)) are only valid in the rest frame comoving with the system. This may be understood since we derived the Fokker-Planck terms $A_{\|}$and $A_{\perp}$ using adiabatic invariants depending on the partial pressures $p_{\|}$and $p_{\perp}$. Conventionally, these partial pressures are taken in the "natural" rest frame that is comoving with the plasma, since the integral

$\left\langle w^{2}\right\rangle=\int \mathrm{d} w w^{2} f(w)$

is not invariant under a coordinate transformation of the form $w \rightarrow w+U$. Now, this specific reference frame is characterised by the fact that the velocity moment $\langle\boldsymbol{w}\rangle$ vanishes. Taking our specific choice of the velocity coordinate system, this translates into the requirements that

$\langle\boldsymbol{w}\rangle=\left\langle\begin{array}{c}w_{\|} \\ w_{\perp} \cos \phi \\ w_{\perp} \sin \phi\end{array}\right\rangle \stackrel{!}{=} \mathbf{0}$,

where the second and third terms vanish because the distribution function $f$ does not depend upon the gyroangle $\phi$. The only nontrivial part of this equation is $\left\langle w_{\|}\right\rangle=0$.

In the absence of stochastic processes, any single point in phase space will remain forever a single point, which justifies the approach

$f_{2}\left(w_{\|}, w_{\perp}\right)=f_{1}\left(\bar{w}_{\|}(\boldsymbol{w}), \bar{w}_{\perp}(\boldsymbol{w})\right)$, i.e. the statistical weights $f(\boldsymbol{w})$ at the individual points in velocity space are simply moved around, but not smeared out. Here, the subscripts 1 and 2 denote the upstream and downstream distribution functions, respectively. Next, we take into account that the absolute normalisation of the distribution function in an accelerated rest frame is not constant, which is conventionally parameterised using the MHD compression ratio $x$, i.e. $\rho_{2}=x \rho_{1}$. Then, we write down this relation using the full distribution functions,

$$
\begin{aligned}
\rho_{2} & =\int \mathrm{d}^{3} \bar{w} D f_{1}\left(\bar{w}_{\|}, \bar{w}_{\perp}\right) \\
& \stackrel{!}{=} x \rho_{1}=x \int \mathrm{d}^{3} w f_{1}\left(w_{\|}, w_{\perp}\right),
\end{aligned}
$$

where $x$ is an arbitrary positive number that has to be derived from the MHD jump conditions. Since this relation must not depend on the $f_{i}$ or $x$, we see that

$\mathrm{d}^{3} \bar{w} D \stackrel{!}{=} \mathrm{d}^{3} w x$

In other words, the Jacobi determinant of the transformation must encode the compression ratio between the upstream and downstream sides in a specific way. This result means that $D$ may not depend upon the particle velocities, and the relation connecting upstream and downstream variables must therefore be linear, such as

$$
\begin{aligned}
w_{\|} & =C_{\|} \bar{w}_{\|}+C_{\|}^{\prime} \bar{w}_{\perp} \\
w_{\perp} & =C_{\perp}^{\prime} \bar{w}_{\|}+C_{\perp} \bar{w}_{\perp} .
\end{aligned}
$$

Now, all that remains to do is to determine the form of the coefficients $C_{i}$ and $B_{i}$. To do this, we take Eq. (35) on the downstream side and express it in the integral form,

$$
\begin{aligned}
0 & \stackrel{!}{=} \int \mathrm{d}^{3} w w_{\|} f_{2}\left(w_{\|}, w_{\perp}\right) \\
& =\int \mathrm{d}^{3} w w_{\|} f_{1}\left(\bar{w}_{\|}(\boldsymbol{w}), \bar{w}_{\perp}(\boldsymbol{w})\right) \\
& =\int \mathrm{d}^{3} \bar{w} D\left(C_{\|} \bar{w}_{\|}+C_{\|}^{\prime} \bar{w}_{\perp}\right) f_{1}\left(\bar{w}_{\|}, \bar{w}_{\perp}\right) \\
& =0+B_{\|} \int \mathrm{d}^{3} \bar{w} D f_{1}\left(\bar{w}_{\|}, \bar{w}_{\perp}\right)
\end{aligned}
$$

Here, the first term vanishes because of our choice of the reference frame, while the second integral is always greater than zero. Therefore, to make the entire expression vanish, one requires $C_{\|}^{\prime}=0$, i.e. the parallel velocities do not become mixed with the perpendicular ones. Using Eq. (38) and writing out the differential then allows to prove that the perpendicular velocities do not get parallel contributions either. This means that the most general, mass flow conserving transformation between the upstream and downstream coordinates is given by

$$
\begin{aligned}
w_{\|} & =C_{\|} \bar{w}_{\|} \\
w_{\perp} & =C_{\perp} \bar{w}_{\perp} .
\end{aligned}
$$

Then, using Eq. (38), one immediately sees that

$\mathrm{d}^{3} \bar{w} D=\mathrm{d}^{3} w C_{\|} C_{\perp}^{2}=\mathrm{d}^{3} w x$

where the additional factor $C_{\perp}$ follows from the fact that $\mathrm{d}^{3} w \propto$ $w_{\perp}$ in cylinder coordinates. In other words, we obtain the additional condition

$C_{\|} C_{\perp}^{2}=x$ 
Now, taking Eqs. (36) and the most general coordinate transformation (i.e. Eqs. (42) and (43)), we see that the most general, mass flow conserving solution of Eq. (1) is given by

$f_{2}\left(w_{\|}, w_{\perp}\right)=f_{1}\left(\frac{w_{\|}}{C_{\|}}, \frac{w_{\perp}}{C_{\perp}}\right)$,

where the parameters $C_{i}$ must be independent of $\boldsymbol{w}$.

From this point, the rest of our formalism is rather straightforward mathematics. Taking Eqs. (42) and (43), inserting them into Eq. (1) and comparing coefficients then allows to reduce the partial differential equation to the two ordinary differential equations

$\frac{\mathrm{d} C_{\|}}{\mathrm{d} s}=-\frac{A_{\|}}{w_{\|}} C_{\|}$

and

$\frac{\mathrm{d} C_{\perp}}{\mathrm{d} s}=-\frac{A_{\perp}}{w_{\perp}} C_{\perp}$.

Since the coefficients $C_{i}$ must not depend upon $w$, the FokkerPlanck terms $A_{i}$ must be linear functions of $w_{i}$,

$A_{i}\left(w_{i}, s\right)=a_{i}(s) w_{i}$,

and the differential equations are formally solved by

$C_{i}=\exp \left(-\int a_{i} \mathrm{~d} s\right)$

Then, the Eqs. (49), (50) and (45), applied in this order, allow us to determine if a kinetic equation is able to describe an MHD shock while conserving the mass flow, and the downstream distribution function is given by Eq. (46).

Now, we may apply this formalism to the equation that we derived in this study. Obviously, the $A_{i}$ given by Eqs. (31) and (32) fulfill Eq. (49), i.e. they are linear functions of their corresponding velocities. Since they are of the form $\mathrm{d} / \mathrm{d} s \ln g(s)$, evaluating Eq. (50) is trivial as well because the exponential and logarithmic functions cancel out, leading to

$C_{\|}=x \frac{B_{1}}{B_{2}}$

and

$C_{\perp}=\sqrt{\frac{B_{2}}{B_{1}}}$,

from which we automatically see that Eq. (45) is indeed fulfilled. We may also derive analytic relations between all upstream and downstream MHD moments in the plasma rest frame, which are equivalent to knowledge of the distribution function. According to Siewert \& Fahr (2007b), this relation is given by

$a_{i j, 2}=\left\langle w_{\|}^{i} w_{\perp}^{j}\right\rangle_{2}=\left(C_{\|} C_{\perp}^{2}\right) C_{\|}^{i} C_{\perp}^{j} a_{i j, 1}=x C_{\|}^{i} C_{\perp}^{j} a_{i j, 1}$.

The downstream pressure anisotropy derived using this formalism is then

$\lambda_{2}=\frac{C_{\perp}^{2}}{C_{\|}^{2}} \lambda_{1}=\left(\frac{B_{2}}{B_{1}}\right)^{3} x^{-2} \lambda_{1}$.

For the parallel shock $\left(\Theta_{B \mathrm{n}}=0\right)$, this relation simplifies to

$\lambda_{2, \|}=\frac{\lambda_{1}}{x^{2}}$,
Table 1. Initial upstream and final downstream parameters for a singlefluid, ion-only plasma. The downstream pressure anisotropies $\lambda$ are not estimated, but are an exact result using Eq. (54). Normalised values are used.

\begin{tabular}{ccc}
\hline \hline & Upstream & Downstream \\
& Perpendicular shock \\
\hline$\rho, x$ & 1 & 2.0078 \\
$U_{\mathrm{n}}$ & $10^{5}$ & $4.9805 \times 10^{4}$ \\
$\lambda$ & 1 & 2.0078 \\
$p_{\|}$ & 0.01 & 0.066 \\
$p_{\perp}$ & 0.01 & 0.133 \\
\hline \multicolumn{3}{c}{ Inclined $\left(\Theta_{B \mathrm{n}}=45^{\circ}\right)$} \\
$\rho, x$ & 1 & 2.137 \\
$U_{\mathrm{n}}$ & $10^{5}$ & $4.679 \times 10^{4}$ \\
$\lambda$ & 1 & 1.718 \\
$p_{\|}$ & 0.01 & 0.107 \\
$p_{\perp}$ & 0.01 & 0.183 \\
\hline Almost parallel shock $\left(\Theta_{B \mathrm{n}}=1^{\circ}\right)$ \\
$\rho, x$ & 1 & 2.253 \\
$U_{\mathrm{n}}$ & $10^{5}$ & $4.4370 \times 10^{4}$ \\
$\lambda$ & 1 & 0.1971 \\
$p_{\|}$ & 0.01 & 0.567 \\
$p_{\perp}$ & 0.01 & 0.112 \\
\hline \multicolumn{3}{c}{}
\end{tabular}

while for the perpendicular shock $\left(\Theta_{B \mathrm{n}}=\pi / 2\right)$, where one obtains $B_{2}=x B_{1}$ (Erkaev et al. 2000), the downstream pressure anisotropy is given by

$\lambda_{2, \perp}=x \lambda_{1}$

Obviously, the kinetic behaviour of an arbitrary distribution function across the shock depends on only two parameters, the MHD compression ratio $x$ and the total magnetic field strength ratio $B_{2} / B_{1}$, i.e. on one parameter related to the massive particles, and one parameter related to the electromagnetic fields. It is noteworthy that the behaviour of the distribution function depends only on upstream and downstream quantities, and not on the fine structure of the shock itself. In conventional MHD, it is usually assumed that a few velocity moments of low order are sufficient to describe the behaviour of the system; in this light, our result may be interpreted in a way that, at least when considering shocks, these few lowest-order moments may be further boiled down to a single kinetic parameter. This result likewise hints that our kinetic equation successfully describes the nonMHD region of the shock in a quasi-MHD approximation, which is as close to MHD as possible, while leaving a sufficient number of degrees of freedom to include strictly non-MHD behaviour. We return to this point in a future study.

Since this formalism was derived in the comoving reference frame of the plasma, which must always exist no matter how complicated the microphysics in the system may be, we call this the minimal kinetic extension. In other reference frames, this extension should be similarly applicable, although the transformation of the velocity moments between the different reference frames is mathematically complicated, and not pursued further in this study.

Using the treatment of the MHD shock presented in this paper, we show a few selected results. We represent the upstream distribution function using a bi-Maxwellian function,

$f(\boldsymbol{w})=\exp \left(-\frac{w_{\|}^{2}}{\theta_{\|}^{2}}-\frac{w_{\perp}^{2}}{\theta_{\perp}^{2}}\right)$, 
with

$\theta_{i}=\frac{2 k T_{i}}{m_{p}}=\sqrt{\frac{2 p_{i}}{\rho}}$,

where the second identity follows from the ideal gas equation. Then, we use the same upstream parameters as those used by Erkaev et al. (2000), i.e. the Alfvenic Mach number $M_{\mathrm{A}}=2$ and the sonic parameter $A_{\mathrm{s}}=0.01$. Using these parameters, it is possible to derive a more conventional form of the upstream parameters, the magnetic field strength

$B=\frac{\sqrt{4 \pi \rho_{1} U_{1}^{2}}}{M_{\mathrm{a}}}$

and the upstream perpendicular pressure,

$p_{\perp}=A_{\mathrm{s}} \rho_{1} U_{1}^{2}$.

In addition to the initial two dimensionless parameters, we use a more conventional approach to the upstream mass flow, by adopting the mass density $\rho_{1}=1 \mathrm{~m}^{-3}$ and the upstream bulk velocity $U=U_{\mathrm{n}}=10^{5} \mathrm{~m} / \mathrm{s}$. Since the solution of the anisotropic jump conditions does not depend on the mass flow $U_{\mathrm{n}} \rho$ (see, e.g. Erkaev et al. 2000; Vogl et al. 2003), any other choice of the parameters constituting the mass flow would lead to similar results. In contrast to this, we remark that the dimensionless parameters used earlier would influence the result. Finally, we assume that, on the upstream side, the plasma is perfectly isotropic, i.e. $\lambda_{1}=1$, and that the magnetic field orientation with respect to the shock normal is characterised by the angle $\Theta_{B n}$. For convenience, these upstream parameters are collected in Table 1.

Next, we solve the anisotropic MHD jump conditions (Hudson 1970; Erkaev et al. 2000) for the upstream parameters given. In a classical (i.e. MHD-only) approach to this problem, the equations are underdetermined, and one is faced with one more downstream parameter than equations, which means that an additional equation must be derived using a different formalism. In this study, we follow Erkaev et al. (2000) and select the downstream pressure anisotropy $\lambda_{2}$, which may be described using Eq. (54). Using this approach, we finally arrive at a unique solution for the downstream parameters, which is given in Table 1. Using this solution, we derive the MHD compression ratio $x$ and the "field compression ratio" $B_{2} / B_{1}$, allowing us to determine the full distribution function on the downsteam side.

Since the perpendicular shock has already been treated in Siewert \& Fahr (2007a), and the current approach to the force terms leads to identical results, we focus on the inclined and parallel shocks. In Figs. 1 and 2, we present cuts through the distribution functions for an inclined shock $\left(\Theta_{B \mathrm{n}}=45^{\circ}\right)$ on the upstream and downstream sides, at $w_{\perp}=0$ and $w_{\|}=0$, respectively. These figures demonstrate a basic property of our solution (Eq. (46)), namely that the shock does not modify the basic shape of the distribution function, which is still of the characteristic Maxwellian form. Instead, it modifies the broadness of this distribution, i.e. the parameter $\theta_{i}$ in Eq. (57), which is a function of the partial pressures. For the inclined shock, both components of the velocity are modified, which directly follows from the fact that, in this case, both coefficients $C_{i}$ are not unity (i.e. in Eqs. (51) and (52), the magnetic field ratio is not 1 or $x$ ).

For the parallel shock, the situation is different. Since this approach is defined by $B=B_{\mathrm{n}}$, and it follows from the MHD jump conditions that the normal magnetic field is conserved, the magnetc field ratios appearing in the coefficients $C_{i}$ are unity, and

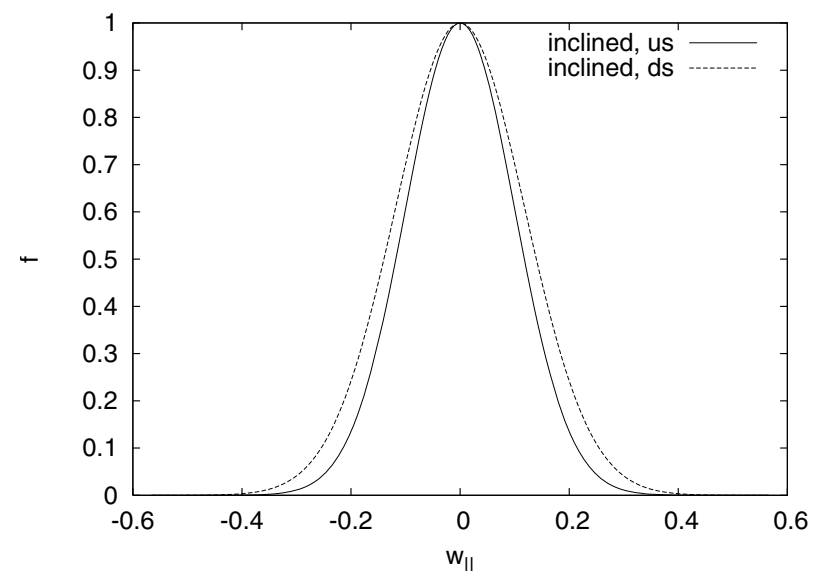

Fig. 1. Representative cuts through the distribution function at $w_{\perp}=0$ for the inclined shock, using an upstream bi-Maxwellian distribution function and the parameters from Table 1.

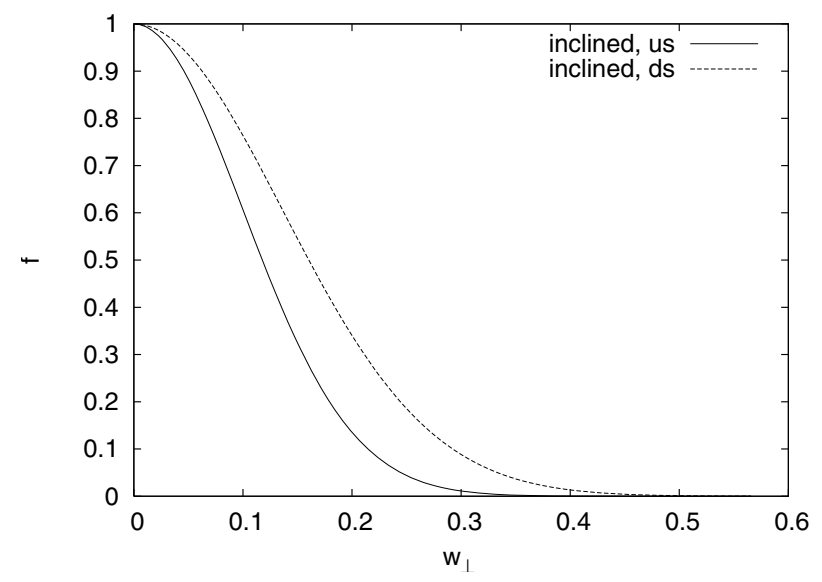

Fig. 2. Representative cuts through the distribution function at $w_{\|}=0$ for the inclined shock, using an upstream bi-Maxwellian distribution function and the parameters from Table 1.

$C_{\perp}=1$, which leads to a completely unmodified perpendicular velocity. This effect is demonstrated in Figs. 3 and 4, where we present, again, cuts through the upstream and downstream distribution functions for an (almost) parallel shock. This is similar to the earlier result obtained for the perpendicular shock (Siewert \& Fahr 2007a), where the parallel velocity components remain untouched.

Finally, we point out that Eq. (33) does not depend upon the behaviour of the MHD quantities inside the transition region, which is in excellent agreement with MHD, and which also implies that we indeed find a kinetic description for the MHD shock that depends essentially only on MHD quantities. However, we emphasize that this approach is, by no means, a globally complete description, but applies only under the restrictions imposed by the MHD approach to the shock. First of all, MHD requires that the electromagnetic fields are frozenin, i.e. convected along with the background plasma. Without this requirement, there would be no motion perpendicular to the magnetic field lines, and no perpendicular shock either. Therefore, although the frozen-in field condition is derived within the framework of classical MHD, it must be valid even when all other MHD requirements fail, since otherwise, there would be no perpendicular shock. For this reason, it must be expected that the fields are still frozen-in into the system even in 


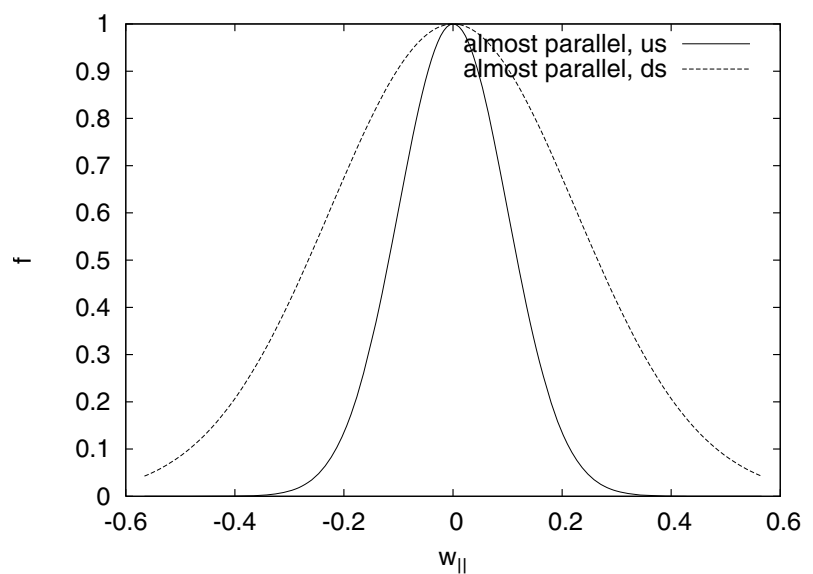

Fig. 3. Representative cuts through the distribution function at $w_{\perp}=$ 0 for the almost parallel shock $\left(\Theta_{B \mathrm{n}} \simeq 1^{\circ}\right)$, using an upstream biMaxwellian distribution function and the parameters from Table 1.

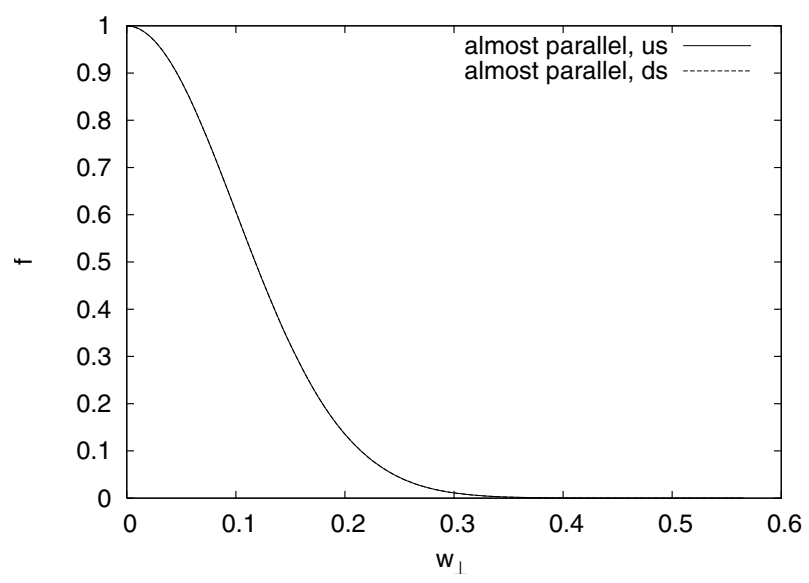

Fig. 4. Representative cuts through the distribution function at $w_{\|}=$ 0 for the almost parallel shock $\left(\Theta_{B \mathrm{n}} \simeq 1^{\circ}\right)$, using an upstream biMaxwellian distribution function and the parameters from Table 1.

the transition layer of the shock, in the sense of a generalised frozen-in field condition.

In addition, the MHD approach to shocks requires that the system is charge-neutral, i.e. that there are no local electric currents present. However, in a more consistent description, one has to include both ions and electrons as separate, interacting fluids. On the other hand, the jump conditions are explicitly tailored to one single fluid, which is typically interpreted in terms of an ion flow, with the implicit asumption that the much lighter electrons are convected along with the rest of the system, and that quasineutrality is obtained. Therefore, to arrive at a more consistent description of the MHD shock, one requires a two-fluid generalization of the MHD jump conditions, including an MHD formulation of charge-neutrality on the upstream and downstream sides. This, however, opens yet another problem, namely the fact that, inside the transition region, where MHD is not applicable, quasineutrality may no longer be an absolute requirement. Considering that the electron distribution function may be quite different from the ion one, this is clearly not a trivial problem. We emphasize that even the particle-field interactions present in MHD may already be interpreted as a two-fluid system, with one fluid being composed of massive particles, and the other fluid of frozen-in fields. In light of this interpretation, interactions between multiple fluids should, in fact, be possible in the framework of MHD. Such an approach would allow to describe wave generation, by including the wavemodes as yet another separate fluid. On the kinetic level, such interactions between various components of the model is realised by upgrading the Boltzmann-Vlasov equation to a Fokker-Planck-like form, where the interactions are parameterised as diffusion coefficients. To the best of our knowledge, there exists no comparable systematic theory of interacting fluids on the MHD level yet. The closest thing to such a theory found in literature is two-fluid hydrodynamics (see Holzer \& Axford 1970).

Clearly, a self-consistent solution to all of these problems may become complicated and is far beyond the scope of this present study. As a first step towards such a description, we are currently working on a multifluid generalisation of the classical MHD jump conditions. Although this work is close to completion, we point out that the "initial problem", i.e. the fact that the anisotropic jump conditions are not perfectly closed, appears to be only the literal tip of the iceberg; for multiple fluids, the amount of free parameters seems to be growing, which offers an excellent interface to include fluid-fluid interactions, in terms of additional conditions required to close the generalised jump conditions. In face of all these aspects, our current result must be interpreted as a working, self-consistent description of the classical, single-fluid MHD shock only, and as a basis for future work.

\section{Applications and outlook}

\subsection{On the incompleteness of a single-fluid system}

Taking Eq. (53), we see that the partial downstream pressures are given by

$p_{\|, 2}=x C_{\|}^{2} p_{\|, 1}$

and

$p_{\perp, 2}=x C_{\perp}^{2} p_{\perp, 1}$.

As demonstrated by Erkaev et al. (2000), the anisotropic MHD jump conditions are underdetermined, and one additional equation is required, which they associated with the downstream pressure anisotropy $\lambda_{2}$. Now, however, the minimal kinetic extension gives us two additional equations, transforming the underdetermined system of equations into an overdetermined one. This follows from the fact that the downstream pressure anisotropy is defined by

$\lambda_{2}=\frac{p_{\perp, 2}}{p_{\|, 2}}$,

which is invariant under the transformation

$p_{\|, \perp}^{\prime}=C \cdot p_{\|, \perp}$,

indicating that the absolute normalisation of the pressures is not preserved by $\lambda_{2}$, and that, taking $\lambda_{2}$ alone might result in a solution that does not satisfy Eqs. (61) and (62). Introducing the parameter (Erkaev et al. 2000)

$\epsilon=1-4 \pi \frac{p_{\|}-p_{\perp}}{B^{2}}$

one may express most of the partial pressure terms in the jump conditions as a function of $\epsilon$ and $\lambda_{2}$, with one isolated perpendicular pressure remaining, which enables us to derive one of the partial pressures from MHD, and leads to two determination conditions for $p_{\perp, 2}$. However, $\epsilon$ is invariant under Eq. (64), since such a renormalisation may also be interpreted in terms of 
$B^{\prime 2} \rightarrow C \cdot B^{2}$. This is sufficient to prove that Erkaev et al. (2000) are unable to predict the correct normalisation of the partial pressures, and that just providing an expression for $\lambda_{2}$ alone is insufficient to arrive at a closed system of equations. This may be understood since any theory capable of predicting the downstream pressure anisotropy must also be able to predict both individual partial pressures, which results in two more equations instead of just one, replacing the previously underdetermined system of equations with an overdetermined system. A possible solution for this situation is the inclusion of electrons, which introduces additional equations and parameters that might lead to a more consistent description. Based on our current description of a single fluid shock, we are currently working on a consistent description of a multifluid shock, which may be used to explicitly model quasineutrality and possibly also stochastic interactions or hybrid fluid-particle descriptions in a more systematic way than commonly found in literature.

\subsection{The parallel shock and the transition region}

Taking the general anisotropic jump conditions (Erkaev et al. $2000)$ and specialising them to the parallel shock $\left(B_{\mathrm{t}}=0, B_{\mathrm{n}}=\right.$ const.), one obtains

$$
\begin{aligned}
{\left[\left[B_{\mathrm{n}}\right]\right] } & =0 \\
{\left[\left[\rho U_{\mathrm{n}}\right]\right] } & =0 \\
{\left[\left[U_{\mathrm{t}} B_{\mathrm{n}}\right]\right] } & =0 \\
{\left[\left[p_{\|}+\rho U_{\mathrm{n}}^{2}\right]\right] } & =0 \\
{\left[\left[\rho U_{\mathrm{n}} U_{t}\right]\right] } & =0 \\
{\left.\left.\left[U_{\mathrm{n}}^{2}\right)\right]\right] } & =0,
\end{aligned}
$$

Obviously, this relation does not contain any variable magnetic field terms, which may be interpreted in terms of the plasma following the field lines. In other words, pure MHD is unable to describe a parallel shock, and inside the transition region of the shock, pure MHD must no longer hold. The existence of a parallel shock is mostly accepted on the condition that many astrophysical shock configurations (see e.g. Treumann \& Scholer 2002) do require this configuration. From a mathematical point of view, the parallel shock may be described in terms of the limit $\Theta_{B \mathrm{n}} \rightarrow 0^{\circ}$. As it turns out, the downstream transversal magnetic field does not converge towards zero in this limit, and the perfectly parallel shock may be unphysical, being replaced instead with a shock where the magnetic field is parallel on the upstream side, but not on the downstream side. However, this approach requires considerably more work, as the conventional anisotropic Rankine-Hugoniot equations do not allow such a solution.

While, in principle, many interpretations of this behaviour are possible, perhaps the most straightforward idea is that the transition region of the MHD shock differs from ideal MHD predictions, and that some of the jump conditions have to be modified. Since energy and momentum are conserved quantities even outside of MHD, and the normal magnetic field conservation is related to many other, non-MHD plasmaphysical applications as well, the conservation of the transverse electric field is the only MHD jump conditions which may, perhaps, be modified by the shock. This jump condition is closely related to the so-called frozen-in field condition (Alfvén \& Fälthammar 1963),

$\partial_{\mathrm{t}} \boldsymbol{B}-\boldsymbol{\nabla} \times(\boldsymbol{U} \times \boldsymbol{B})=0$, which is required to allow motion perpendicular to the magnetic field, as it is allowed in conventional MHD. Clearly, the time-dependent term, which is usually set to zero on the far upstream and downstream sides, must be nonzero inside the shock to preserve frozen-in fields. Since the presence of timedependent terms in electrodynamical equations is usually interpreted in terms of plasma waves, this automatically hints that an MHD shock is a natural plasma wave generator. However, since plasma waves usually require a quasineutral system (i.e. where ions and electrons are present in equivalent quantities), one needs to extend the MHD jump conditions to include at least two particle flows (or, alternatively, two charge flows). As already mentioned, we are working on a consistent description of all these aspects. We expect that this description is able to describe the behaviour of the magnetic fields inside the transition region, shedding more light on the currently unsolved points related to the second CGL invariant.

\section{Conclusions}

In this study, we derived an improved version of an earlier kinetic Boltzmann equation derived by Fahr \& Siewert (2006), which attempts to describe MHD shocks, such as the solar wind termination shock. Using a more strict approach in terms of reference frames and initial assumptions, we were able to eliminate the restrictions which emerged in the earlier studies, strengthening the connection between MHD and kinetic theory. This new equation fulfils the requirement derived by Siewert \& Fahr (2007b) based on the conservation of the mass flow, which suggests that Eq. (33) is a self-consistent description of a basic, turbulence-free MHD shock that depends only on MHD upstream and downstream quantities, but not on the behaviour of the plasma in the transition region.

In addition, we derived what might turn out to be a new theory of per-particle invariants, derived from MHD invariants, generalising the well-known equivalence between the single-particle magnetic moment conservation and the equivalent MHD adiabatic invariant. While we have not yet been able to prove that this generalisation leads to physical expressions for all possible MHD invariants, we have found several arguments that strongly suggest that this approach works, at least, for the two adiabatic invariants appearing in the CGL theory. Our current work hints that the conservation of the second CGL invariant is related to a bulk velocity gradient parallel to $\boldsymbol{B}$ and the corresponding reaction of the frozen-in magnetic field (Fahr \& Siewert 2008).

Acknowledgements. We are grateful for financial support to the DFG within the frame of the DFG-Project Fa 97/31-2.

\section{Appendix A: Transformation of an MHD invariant in a per-particle invariant}

In this appendix, we prove that Eq. (22) is always fulfilled, for arbitrary distribution functions $f_{1}(w)$. Writing down the temporal derivative of this expression, one obtains the following requirement

$$
\int \mathrm{d}^{3} w\left(\left(\frac{\mathrm{d}}{\mathrm{d} t} f_{1}(w)\right) w_{\|}^{i} w_{\perp}^{j}+f_{1}(w)\left(\frac{\mathrm{d}}{\mathrm{d} t} w_{\|}^{i} w_{\perp}^{j}\right)\right) \stackrel{!}{=}
$$

$$
\int \mathrm{d}^{3} w f_{1}(w)\left(\frac{\mathrm{d}}{\mathrm{d} t} w_{\|}^{i} w_{\perp}^{j}\right)
$$


In other words, we require

$$
\int \mathrm{d}^{3} w\left(\frac{\mathrm{d}}{\mathrm{d} t} f_{1}(w)\right) w_{\|}^{i} w_{\perp}^{j} \stackrel{!}{=} 0 .
$$

To prove this, we begin by using the most simple distribution function,

$f(\boldsymbol{w})=\delta\left(\boldsymbol{w}-\boldsymbol{w}_{0}\right)$,

which describes a single particle (or a single "cell" in velocity space). Inserting this into Eq. (A.2) leads to

$\int \mathrm{d}^{3} w\left(\frac{\mathrm{d}}{\mathrm{d} t} \delta\left(\boldsymbol{w}-\boldsymbol{w}_{0}\right)\right) w_{\|}^{i} w_{\perp}^{j} \stackrel{!}{=} 0$.

Here, the derivative of the delta function trivially vanishes because of

$$
\begin{aligned}
\frac{\mathrm{d}}{\mathrm{d} t} \int \mathrm{d}^{3} w \delta\left(\boldsymbol{w}-\boldsymbol{w}_{0}\right) w_{\|}^{i} w_{\perp}^{j} & =\frac{\mathrm{d}}{\mathrm{d} t} w_{\|, 0}^{i} w_{\perp, 0}^{j} \\
& =\int \mathrm{d}^{3} w \delta\left(\boldsymbol{w}-\boldsymbol{w}_{0}\right) \frac{\mathrm{d}}{\mathrm{d} t} w_{\|}^{i} w_{\perp}^{j} .
\end{aligned}
$$

This automatically means that

$\int \mathrm{d}^{3} w\left(\frac{\mathrm{d}}{\mathrm{d} t} \delta\left(\boldsymbol{w}-\boldsymbol{w}_{0}\right)\right) w_{\|}^{i} w_{\perp}^{j}=0$.

For a system with $N$ particles, the situation is more complicated. Here, the distribution function is given by

$f_{1}(\boldsymbol{w})=\sum_{n=1}^{N} \alpha_{\mathrm{n}} \delta\left(\boldsymbol{w}-\boldsymbol{w}_{0, n}\right)$,

where

$\sum_{n=1}^{N} \alpha_{\mathrm{n}}(t)=1$

and

$\frac{\mathrm{d}}{\mathrm{d} t} \sum_{n=1}^{N} \alpha_{\mathrm{n}}(t)=0$.

Inserting this distribution function into Eq. (A.2) leads to

$$
\begin{aligned}
0 \stackrel{!}{=} & \sum_{n=1}^{N}\left[\left(\frac{\mathrm{d}}{\mathrm{d} t} \alpha_{\mathrm{n}}(t)\right) \int \mathrm{d}^{3} w \delta\left(\boldsymbol{w}-\boldsymbol{w}_{\mathrm{n}}\right) w_{\|}^{i} w_{\perp}^{j}\right. \\
& \left.+\alpha_{\mathrm{n}}(t) \int \mathrm{d}^{3} w\left(\frac{\mathrm{d}}{\mathrm{d} t} \delta\left(\boldsymbol{w}-\boldsymbol{w}_{\mathrm{n}}\right)\right) w_{\|}^{i} w_{\perp}^{j}\right] .
\end{aligned}
$$

Here, the second term vanishes because of Eq. (A.6). The first term may be removed by noting that, when controlled by the collisionless Boltzmann-Vlasov equation, individual particles in a physical system do always follow determined trajectories, and that therefore the weight functions $\alpha_{\mathrm{n}}(t)$ are constant parameters on these trajectories as a consequence of Liouvilles theorem. For this reason, the first term also vanishes, and we have proven that any temporal change of a velocity moment in an $N$-particle system may be described using the sum of changes of the individual particles.

This approach works only in the absence of stochastical processes, which destroy the uniqueness of the particle trajectories. For this reason, it may be assumed that this approach works for a broad distribution function only when stochastical processes are still absent, as it is the case for the Boltzmann-Vlasov equation. We will present a more detailed analysis under which conditions this holds in a future publication.

\section{References}

Alfvén, H., \& Fälthammar, C. G. 1963, Cosmical Electrodynamics, 2nd Ed. (Oxford: Clarendon Press)

Burlaga, L. F., Ness, N. F., \& Acuna, M. H. 2006a, ApJ, 642, 584

Burlaga, L. F., Ness, N. F., \& Acuna, M. H. 2006b, Geophys. Res. Lett., 33, L21106

Cercignani, C. 1988, The Boltzmann Equation and Its Applications (New York: Springer-Verlag)

Chalov, S. V., \& Fahr, H. J. 1998, A\&A, 335, 746

Chew, G. F., Goldberger, M. L., \& Low, F. E. 1956, Proc. R. Soc. London A, 236,112

Cummings, A. C., Stone, E. C., McDonald, F. B., Heikkila, B. C., \& Lal, N. 2006, in Physics of the inner Heliosheath, AIP Conf. Proc., 858, 86

Decker, R. B., Krimigis, S. M., Roelof, E. C., et al. 2005, Science, 309, 2020

Erkaev, N. V., Vogl, D. F., \& Biernat, H. K. 2000, J. Plasma Physics, 64, 561

Escoubet, C. P., Schmidt, R., \& Goldstein, M. L. 1997, Space Sci. Rev., 79, 11

Fahr, H.-J., \& Siewert, M. 2006, A\&A, 458, 13

Fahr, H.-J., \& Siewert, M. 2007, ASTRA, 3, 21

Fahr, H.-J., \& Siewert, M. 2008, A\&A, 484, L1

Fisk, L. A., \& Gloeckler, G. 2006, ApJ, 640, L79

Hada, T., Onishi, M., Lembege, B., \& Savoinin, P. 2003, J. Geophys. Res., 108, 1233

Holzer, T. E., \& Axford, W. I. 1970, ARA\&A, 8, 31

Hudson, P. D. 1970, Planet. Space Sci., 18, 1611

Kulsrud, R. M. 1983, in Handbook of Plasma Physics, ed. M. N. Rosenbluth, \&

R. Z. Sagdeev (Amsterdam, North-Holland: Elsevier), 1, 115

Lobzin, V. V., Krasnoselskikh, V. V., Josqued, J.-M., et al. 2007,

Geophys. Res. Lett., 34, L05107

Parker, E. N. 1965, Plan. Sp. Sc., 13, 9

Press, W. H. 1987-2002, Numerical recipes (New York: Cambridge University Press)

Schlickeiser, R. 1989, ApJ, 336, 243

Schlickeiser, R. 2002, Cosmic Ray Astrophysics (Berlin: Springer Verlag)

Scholer, M., Shinohara, I., \& Matsukiyo, S. 2003, J. Geophys. Res., 108, 1014

Siewert, M., \& Fahr, H.-J. 2007a, A\&A, 463, 799

Siewert, M., \& Fahr, H.-J. 2007b, A\&A, 471, 7

Stone, E. C., Cummings, A. C., McDonald, F. B., et al. 2005, Science, 309, 2017

Treumann, R. A., \& Scholer, M. 2002, in The Century of Space Science (Norwell: Kluwer Academic Publishers), 1495

Vogl, D. F., Langmayr, D., Erkaev, N. V., et al. 2003, Planet. Space Sci., 51, 715 